score, BMI, serum albumin or prealbumin levels, or adverse event rates were observed, and the investigators concluded that there were no advantages to adding IDPN to oral nutritional supplementation.

Taking all 186 patients together, nutritional status declined markedly in the 6 months before intervention, but a striking and sustained improvement was seen with supplementation. Presence of diabetes was associated with a less-sustained nutritional response and with increased mortality in the second study year. Importantly, a $>30 \mathrm{mg} / \mathrm{l}$ increase in serum prealbumin (a marker of nutritional status) within the first 3 months of supplementation was independently predictive of decreased 2-year mortality and hospitalization rates in those patients without diabetes.

Original article Cano NJM et al. (2007) Intradialytic parenteral nutrition does not improve survival in malnourished hemodialysis patients: a 2-year multicenter, prospective, randomized study. J Am Soc Nephrol 18: 2583-2591

\section{Mortality in hemodialysis patients treated with sevelamer or calcium-based phosphate binders}

Hyperphosphatemia and hypercalcemia are independently associated with an increased risk of cardiovascular calcification and mortality in patients on dialysis. Sevelamer hydrochloride has the potential to reduce serum phosphorus levels without promoting arterial calcification (which can result from use of calcium-based oral phosphate binders [OPBs]) and could contribute to lower all-cause mortality.

The Dialysis Clinical Outcomes Revisited trial randomized 2,103 prevalent hemodialysis patients to sevelamer or a calcium-based OPB; 1,068 patients completed the study (mean treatment time approximately 19 months). Adverse events that resulted in discontinuation were mainly hypercalcemiarelated in the OPB group and gastrointestinal in the sevelamer group.

In the entire group, all-cause and cause-specific (e.g. cardiovascular and infection-related) mortality rates were not significantly different between the two treatments; the study was not, however, adequately powered to detect cause-specific differences. Kaplan-Meier analysis indicated a survival improvement with sevelamer in patients who participated in the trial for 2 years or more (time-treatment interaction; $P=0.02$ ).

Sevelamer was associated with a significant reduction in all-cause mortality in patients over 65 years of age $(P=0.02$ for comparison with OPB treatment); in younger patients, mortality was slightly but not significantly higher with sevelamer. Sevelamer also reduced the frequency of hospitalization among older patients. These findings could be a function of the greater pre-existing calcification burden in older patients on hemodialysis, which could more rapidly facilitate the effects of oral calcium on mortality.

Original article Suki WN et al. (2007) Effects of sevelamer and calcium-based phosphate binders on mortality in hemodialysis patients. Kidney Int 72: 1130-1137

\section{Homocysteine lowering does not improve survival in advanced CKD or ESRD}

High plasma homocysteine levels have been associated with an increased risk for cardiovascular disease, but randomized trials of homocysteine-lowering therapies have reported conflicting results. A recent large, double-blind, randomized controlled trial investigated whether lowering homocysteine levels with folic acid plus the vitamin $\mathrm{B}_{6}$ (pyridoxine) and vitamin $\mathrm{B}_{12}$ (cyanocobalamin) reduced mortality and cardiovascular events in patients with advanced chronic kidney disease (CKD) or end-stage renal disease (ESRD).

Overall, 2,056 adults with either ESRD or advanced CKD (estimated creatinine clearance $\leq 30 \mathrm{ml} / \mathrm{min}$ by the Cockcroft-Gault formula) and a plasma homocysteine level of $\geq 15 \mu \mathrm{mol} / \mathrm{l}$ were randomized to receive either a capsule containing $40 \mathrm{mg}$ folic acid, $100 \mathrm{mg}$ vitamin $\mathrm{B}_{6}$ and $2 \mathrm{mg}$ vitamin $\mathrm{B}_{12}$ (treatment group; $n=1,032$ ) or a placebo capsule (control group; $n=1,024)$ once daily for a median of 3.2 years.

At 3 months, mean homocysteine level had decreased significantly from baseline in the treatment group $(-6.2 \mu \mathrm{mol} / \mathrm{l} ; P<0.001)$, but had not decreased significantly in the control group $(-0.4 \mu \mathrm{mol} / \mathrm{l})$. Treatment did not, however, affect either the primary outcome of all-cause mortality (436 deaths in placebo group vs 448 deaths in the treatment group; 